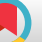

\title{
Anatomical Variations of Accessory Bands in Semitendinosus and Gracilis Tendons Among the Asian Population
}

\author{
Thanathep Tanpowpong ${ }^{1}$, Chaiwat Saengkhiew ${ }^{1}$, Thanasil Huanmanop ${ }^{2}$ and Thun \\ Itthipanichpong, ${ }^{1 *}$ \\ ${ }^{1}$ Department of Orthopedics, King Chulalongkorn Memorial Hospital, Thai Red Cross Society, Chulalongkorn University, Bangkok, Thailand \\ ${ }^{2}$ Department of Anatomy, Chulalongkorn University, Bangkok, Thailand \\ "Corresponding author: Department of Orthopedics, King Chulalongkorn Memorial Hospital, Thai Red Cross Society, Chulalongkorn University, Bangkok, Thailand. Email: \\ thunthedoc@gmail.com
}

Received 2019 January 06; Revised 2019 July 14; Accepted 2019 August 02.

\begin{abstract}
Background: The length and number of accessory bands of gracilis and semitendinosus tendons have high variability among Asian population. Complications during graft harvesting might occur if the surgeon cannot correctly identify these complex structures. Methods: We performed cadaveric knee specimen dissections (level of evidence: level III; descriptive laboratory study (anatomical study)). Each of the accessory bands arising from semitendinosus and gracilis tendons was identified. Size and distance from the origin of accessory bands to the tibial insertion were measured.

Results: Eighty knees were included in the study. For the semitendinosus tendon, we found the following accessory bands in our specimens: no accessory bands in $6 \%$, one band in $56 \%$, and two bands in $38 \%$. The longest distance of an accessory band from tibial insertion was $9.8 \mathrm{~cm}$, with an average distance for the first band of $4.5 \mathrm{~cm}$. The number of accessory bands for the gracilis tendon was, as follows: $0,1,2,3$ and 4 in 20,39, 31, 9, and 1\%, respectively. The longest distance of accessory band was $9.33 \mathrm{~cm}$ from tibial insertion, with an average distance for the first band of $4.7 \mathrm{~cm}$. We found the width of accessory bands in females to be wider than males $(\mathrm{P}=0.0001)$ and the width of accessory bands in right knees to be wider than those in left knees $(\mathrm{P}=0.04)$.

Conclusions: In Asian populations, we found high variability of number of accessory bands from semitendinosus and gracilis tendons. The average distance from the tibial insertion to the first accessory band was $4.5 \mathrm{~cm}$. None of the bands arised more than 10 $\mathrm{cm}$ from the tibial insertion.
\end{abstract}

Keywords: ACL Reconstruction, Semitendinosus, Gracilis, Hamstring, Accessory Bands, Asians

\section{Background}

Ligament reconstruction of the knees is being increasingly performed. Hamstring tendon autografts are commonly used because of their good mechanical property and low donor site morbidity (1-3). One of the complications is premature rupture of tendon during graft harvesting, which can be prevented by thoroughly identifying all accessory bands arising from these tendons (1-8). If premature rupture of the graft occurrs, the diameter of the graft would be shorter and smaller. The risk of failure will be higher if the graft diameter is less than $8 \mathrm{~mm}$ (9). Two previous studies (Candal-Couto and Deehan (10) and Ivey and Prud'homme (11)) showed that the most proximal accessory band was attached to the semitendinosus and gracilis tendons beyond $10 \mathrm{~cm}$ from tibial insertion. If the accessory band was far beyond $10 \mathrm{~cm}$ from the tibial insertion, it would be more difficult to identify the band and the chance of premature rupture of the graft would be higher. In contrast to our experience, we had never noticed accessory bands that arose beyond $10 \mathrm{~cm}$ and the recommendation from Frank et al. (12), he had suggested to clear all the adhesions and free the tendon $10 \mathrm{~cm}$ from the tibial insertion, which corresponds to our hypothesis.

We believed that race/ethnicity may be one of the potential factors responsible for these disparate findings since most previous studies have been conducted in the US and Europe.

\section{Objectives}

Our aim was to identify anatomical variations of the accessory bands of hamstring tendons in the Asian cadaveric specimens to prevent the risk of premature tendon rupture. 


\section{Methods}

\subsection{Data Collection}

We examined cadaver knees which were used for teaching medical students at the Department of Anatomy, Faculty of Medicine, Chulalongkorn University, Bangkok, Thailand. Institutional review board approved before preceding the study. Our inclusion criterion was all the cadavers that were used in the teaching year of 2015, and we excluded cadavers with poor or damaged tissue in which measurement cannot be done or may be invalid. Two investigators evaluated the tissue status in every step of dissection. If one out of two investigators noticed that the tissue was not in optimum condition, we discarded that specimen. Data including age, sex, weight and height were collected. Surgical dissection was performed to identify semitendinosus and gracilis tendons from the insertion on the tibial crest and proximal to the musculotendinous junction. All accessory bands were identified from the origin to the structure which they were inserted. We marked the insertion of semitendinosus and gracilis tendons at the tibial crest as reference points with pins and measured the distance from the reference point to the origin of each accessory band with digital caliper (Becker model EC10, Mumbai, India). Width of the accessory bands was also measured at the area of its origin (Figure 1). Each of the two investigators were assigned to measure three times with 1week intervals. The correlation between sex, height, side of the knees, number and width of accessory bands were calculated.

\subsection{Statistical Analysis}

The reliability was calculated using the intraclass correlation coefficient (ICC). Descriptive statistics was presented as mean and standard deviation (SD). Statistical analyses were calculated with the STATA program paired $t$-test was used for mean comparison of normal distributions of continuous variables. Pearson's correlation coefficient was used to demonstrate correlation between individual parameters. The level of statistical significance was $\mathrm{P}<0.05$.

\section{Results}

We included all 80 knees from 40 cadavers in our study. None of the cadavers were excluded in this study.

The ICCs for intra- and inter-rater agreements were $>$ 0.90 for all measurements. The ICCs value of $>0.80$ defined an excellent agreement (13). The average age of the cadavers were 72.8 years. The baseline characteristics were not statistically different between male and female (Table $1)$.

\begin{tabular}{|c|c|c|c|}
\hline & Male $(N=38,47.5 \%)$ & Female $(\mathrm{N}=42,52.5 \%)$ & PValue \\
\hline Age, $y$ & $72.6 \pm 17$ & $72.9 \pm 15.4$ & 0.87 \\
\hline Weight, kg & $63.1 \pm 10$ & $56.4 \pm 11.2$ & 0.54 \\
\hline Height, cm & $166.8 \pm 5.4$ & $156 \pm 6.8$ & 0.66 \\
\hline BMI & $22.6 \pm 2.8$ & $23.2 \pm 3.1$ & 0.73 \\
\hline
\end{tabular}

${ }^{\mathrm{a}}$ Values are expressed as mean $\pm \mathrm{SD}$.

For the semitendinosus tendon, we could not found accessory bands in $6 \%$ of our specimens, one band in $56 \%$ and two bands for $38 \%$. The average distance of the first accessory band was $4.5 \pm 1.5 \mathrm{~cm}$ from tibial insertion, and the longest distance of accessory band was $9.8 \mathrm{~cm}$ from the tibial insertion. The band's insertion sites were gastrocnemius fascia (91\%), tibial periosteum (8\%), and sartorial fascia (1\%). Mean width of the accessory bands was $3.9 \pm 1.7$ $\mathrm{mm}$.

For the gracilis tendon, we could not find accessory bands in $20 \%$, one band in $39 \%$, two bands in $31 \%$, three bands in $9 \%$ and four bands in $1 \%$. The average distance of the first accessory band was $4.7 \pm 1.7 \mathrm{~cm}$ from the tibial insertion, and the longest distance was $9.3 \mathrm{~cm}$ from the tibial insertion. The band's insertion sites were gastrocnemius fascia (74\%), tibial periosteum (22\%), and sartorial fascia (4\%). Mean width of the accessory bands was $3.53 \pm 1.45$ $\mathrm{mm}$.

Regarding gender, we found that accessory bands in females were significantly wider than males $(4.0 \pm 1.8 \mathrm{~mm}$ vs. $3.5 \pm 1.2 \mathrm{~mm}, \mathrm{P}=0.001)$, but the number of accessory bands was not significant between females $(4.9 \pm 2.0)$ vs. male $(5.3 \pm 1.9)(P=0.34)$.

There were also found difference in the width of accessory band in the right knees, as compared to the left knees $(3.9 \pm 1.7 \mathrm{~mm}$ vs $3.6 \pm 1.4 \mathrm{~mm}$, respectively $)(\mathrm{P}=0.04)$. The number of accessory bands found in the right and left knees were not significant $(5.1 \pm 1.9$ vs. $5.2 \pm 1.8$, respectively) $(\mathrm{P}=0.51)$ There may be a trend in the correlation between the height of cadavers and the number of accessory bands $(\mathrm{P}=0.07)$.

\section{Discussion}

Accessory bands arising from semitendinosus and gracilis tendons are commonly found during graft harvesting $(10,14,15)$. Failure to identify these structures can lead to premature amputation of the tendon $(4,6,8,14)$. Understanding the anatomical variations in this area may help in reducing complications related to graft harvest especially premature graft rupture (16). The graft should be at least 

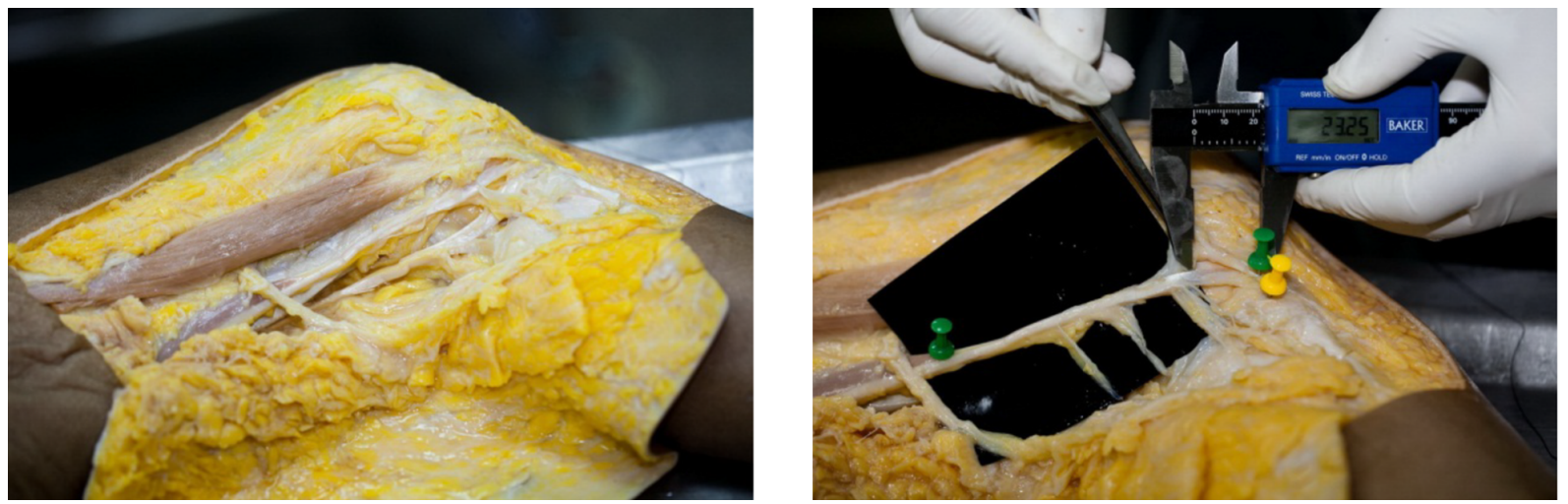

Figure 1. The anatomical dissection and method of measurement

$150-160 \mathrm{~mm}$ in length (75 - $80 \mathrm{~mm}$ loop graft) and $7-8$ $\mathrm{mm}$ in diameter to secure the fixation and avoid risk of graft failure. If the length and the size of the graft were not adequate, alternative autograft or synthetic graft may be used (17). Several studies have mentioned the location of accessory bands. A study by Candal-Couto and Deehan (10) looked at 10 cadaveric knees and found accessory bands arising $>10 \mathrm{~cm}$ in 8 . Reina et al. (18) dissected 30 fresh cadaveric knees and found accessory bands arising $>10 \mathrm{~cm}$ in 3. The only study by Yasin et al. (19) which measured accessory band after harvesting form patients undergoing ligament reconstruction, found that 5 from 25 specimens had accessory band arising $>10 \mathrm{~cm}$.

To our knowledge, this is the first study on anatomical variation of accessory bands of the aforementioned tendons in a substantial number of cadaveric knee specimens in Asians (80 embalmed cadaveric knees). In contrast to the previous study $(10,(18),(19))$ we could not demonstrate accessory bands arising more than $10 \mathrm{~cm}$ from the tibial insertion. Average height and weight of our population were close to the previous work by Reina et al. (18). We postulate that racial difference may play some role because most prior studies were performed in the western population.

Semitendinosus has more constant anatomy. We found only one accessory band in most of our case (56\%) and never found $>2$ bands in any specimen. Unlike a previous paper by Yasin et al. (19) which found 3 bands in most cases.

Gracilis has more anatomical variability. We found either one or two bands in most cases (70\%) but could find 3 - 4 bands in $10 \%$. Twenty percent of gracilis tendons did not have accessory bands, as compared with $6 \%$ in semitendinosus. This high variability of gracilis tendon reminds surgeons to be more careful and look for all possible accessory bands during graft harvesting.
The first accessory band which surgeons may encounter during graft harvesting is about $4.5 \mathrm{~cm}$ from the tibial insertion which is $2 \mathrm{~cm}$ shorter compared to the study in 102 lower limb by Olewnik in Poland. (20) These findings were similar in both semitendinosus and gracilis tendons. Most accessory bands inserted to the gastrocnemius fascia.

We found that the width of accessory band in the right knees were wider than the left knees significantly, which we hypothesized that more use of muscles and tendons on the dominant side might explain this finding since most of the general population were right-sided dominant. We also found that the width of accessory bands in females was wider than males. Biologic plausibility might be difficult to explain this finding but could relate to hormonal difference. The widest accessory band in our study was 9.5 $\mathrm{mm}$ which is wider than the main gracilis tendon itself. Surgeons should pay special attention during surgery not to cut the main tendon rather than accessory bands by determining only by the width.

Our limitation is that most of our cadavers were older than 70 years old that might not reflect the general population who need a knee ligament reconstruction. Due to embalmed cadavers, soft tissue distortion and shrinkage from the process may not reflect the in vivo situation.

\subsection{Conclusions}

In Asian populations, we found high variability of number of accessory bands from semitendinosus and gracilis tendons. The average distance from the tibial insertion to the first accessory band was $4.5 \mathrm{~cm}$. None of the bands arised more than $10 \mathrm{~cm}$ from the tibial insertion. Our findings in our study would help surgeons to understand more about anatomical structure in this ethnicity 
and prevent premature rupture of the hamstring autograft.

\section{Footnotes}

Authors' Contribution: Thanathep Tanpowpong: first author, created idea, measurement and manuscript correction. Chaiwat Saengkhiew: IRB application, data collecting, measurement and manuscript writing. Thanasil Huanmanop: help with knee cadavers checking. Thun Itthipanichpong: Manuscript writing, collected and interpreted data.

Conflict of Interests: The authors have no conflict of interests to disclose.

Ethical Approval: The Institutional Review Board, Faculty of Medicine, Chulalongkorn University, Bangkok, Thailand approved this study in February 2014 for doing research in cadaver subjects.

Financial Disclosure: No disclosure for all of the authors. Funding/Support: There was no funding/support for the study.

\section{References}

1. Chen L, Cooley V, Rosenberg T. ACL reconstruction with hamstring tendon. Orthop Clin North Am. 2003;34(1):9-18. doi: 10.1016/S00305898(02)00016-0. [PubMed: 12735197].

2. Noyes FR, Butler DL, Grood ES, Zernicke RF, Hefzy MS. Biomechanical analysis of human ligament grafts used in knee-ligament repairs and reconstructions. J Bone Joint Surg Am. 1984;66(3):344-52. doi: 10.2106/00004623-198466030-00005. [PubMed: 6699049].

3. Poolman RW, Abouali JA, Conter HJ, Bhandari M. Overlapping systematic reviews of anterior cruciate ligament reconstruction comparing hamstring autograft with bone-patellar tendon-bone autograft: Why are they different? J Bone Joint Surg Am. 2007;89(7):1542-52. doi: 10.2106/JBJS.F.01292. [PubMed: 17606794].

4. Almazan A, Miguel A, Odor A, Ibarra JC. Intraoperative incidents and complications in primary arthroscopic anterior cruciate ligament reconstruction. Arthroscopy. 2006;22(11):1211-7. doi: 10.1016/j.arthro.2006.06.019. [PubMed: 17084299].

5. Charalambous CP, Alvi F, Phaltankar P, Gagey O. Hamstring tendon harvesting-Effect of harvester on tendon characteristics and soft tissue disruption; cadaver study. Knee. 2009;16(3):183-6. doi: 10.1016/j.knee.2008.11.010. [PubMed:19272780].

6. Wittstein JR, Wilson JB, Moorman CT. Complications related to hamstring tendon harvest. Oper Tech Sports Med. 2006;14(1):15-9. doi: 10.1053/j.otsm.2006.02.008.
7. Ferrari JD, Ferrari DA. The semitendinosus: anatomic considerations in tendon harvesting. Orthop Rev. 1991;20(12):1085-8. [PubMed: 1771105].

8. Tuncay I, Kucuker H, Uzun I, Karalezli N. The fascial band from semitendinosus to gastrocnemius: the critical point of hamstring harvesting: An anatomical study of 23 cadavers. Acta Orthop. 2007;78(3):361-3. doi: 10.1080/17453670710013933. [PubMed: 17611850$]$.

9. Magnussen RA, Lawrence JT, West RL, Toth AP, Taylor DC, Garrett WE. Graft size and patient age are predictors of early revision after anterior cruciate ligament reconstruction with hamstring autograft. Arthroscopy. 2012;28(4):526-31. doi: 10.1016/j.arthro.2011.11.024. [PubMed: 22305299].

10. Candal-Couto JJ, Deehan DJ. The accessory bands of Gracilis and Semitendinosus: An anatomical study. Knee. 2003;10(4):325-8. doi: 10.1016/S0968-0160(02)00154-0. [PubMed: 14629934].

11. Ivey M, Prud'homme J. Anatomic variations of the pes anserinus: a cadaver study. Orthopedics. 1993;16(5):601-6. [PubMed: 8327387].

12. Frank RM, Hamamoto JT, Bernardoni E, Cvetanovich G, Bach BR Jr, Verma NN, et al. ACL reconstruction basics: quadruple (4-strand) hamstring autograft harvest. Arthrosc Tech. 2017;6(4):e1309-13. doi: 10.1016/j.eats.2017.05.024. [PubMed: 29354434]. [PubMed Central: PMC5622412].

13. Landis JR, Koch GG. The measurement of observer agreement for categorical data. Biometrics. 1977;33(1):159-74. [PubMed: 843571].

14. Pagnani MJ, Warner JJ, O’Brien SJ, Warren RF. Anatomic considerations in harvesting the semitendinosus and gracilis tendons and a technique of harvest. Am J Sports Med. 1993;21(4):565-71. doi: 10.1177/036354659302100414. [PubMed: 8368418].

15. Warren LF, Marshall JL. The supporting structures and layers on the medial side of the knee: An anatomical analysis. J Bone Joint Surg Am. 1979;61(1):56-62. doi: 10.2106/00004623-197961010-00011. [PubMed: 759437].

16. Charalambous CP, Alvi F, Sutton PM. Management of intraoperative complications in arthroscopic primary anterior cruciate ligament reconstruction. J Knee Surg. 2015;28(2):165-74. doi: 10.1055/s-00341373739. [PubMed: 24752920].

17. McGuire DA, Hendricks SD. Anterior cruciate ligament reconstruc tion graft harvesting: Pitfalls and tips. Sports Med Arthrosc Rev. 2007;15(4):184-90. doi: 10.1097/JSA.0b013e3181595bb6. [PubMed: 18004217].

18. Reina N, Abbo O, Gomez-Brouchet A, Chiron P, Moscovici J, Laffosse JM. Anatomy of the bands of the hamstring tendon: How can we improve harvest quality? Knee. 2013;20(2):90-5. doi: 10.1016/j.knee.2012.06.003. [PubMed: 22766187].

19. Yasin MN, Charalambous CP, Mills SP, Phaltankar PM. Accessory bands of the hamstring tendons: A clinical anatomical study. Clin Anat. 2010;23(7):862-5. doi: 10.1002/ca.21020. [PubMed: 20607820].

20. Olewnik L, Gonera B, Podgorski M, Polguj M, Jezierski H, Topol M. A proposal for a new classification of pes anserinus morphology. Knee Surg Sports Traumatol Arthrosc. 2019;27(9):2984-93. doi:10.1007/s00167018-5318-3. [PubMed: 30535546]. [PubMed Central: PMC6706366]. 